\title{
A systematic review and meta-analysis of Toxoplasma gondii infection among the Mexican population
}

\author{
Ma de la Luz Galvan-Ramirez ${ }^{1 *}$, Rogelio Troyo ${ }^{1}$, Sonia Roman³ , Carlos Calvillo-Sanchez ${ }^{1}$
} and Rosamaria Bernal-Redondo ${ }^{2}$

\begin{abstract}
Background: Toxoplasmosis is a disease caused by Toxoplasma gondii and at least one-third of the world's population has detectable T. gondii antibodies. The seroprevalence of T.gondii ranges from 15\% to 50\% among the Mexican general population. The aim of this work was to determine the mean prevalence and weighted mean prevalence of $T$. gondii infection, and to evaluate the epidemiological transition of infection in Mexico.

Methods: Pub Med, Lilacs, Medline, Latindex, Google Scholar data bases were searched to retrieve reports from 1951 up to 2012 regarding prevalence data, diagnostic tests and risk factors of infection among the adult population. Data collection and criteria eligibility was established in order to determine the crude prevalence (proportion of positive cases) of each study, together with weighted population prevalence according to individual research group categories to limit the bias that may impose the heterogeneous nature of the reports. A Forest Plot chart and linear regression analysis were performed by plotting the prevalence of infection reported from each study over a period of sixty years.

Results: A total of 132 studies were collected from 41 publications that included 70,123 individuals. The average mean prevalence was $27.97 \%$, and weighted mean prevalence was $19.27 \%$. Comparisons among different risk groups showed that the weighted prevalence was higher in women with miscarriages (36.03\%), immunocompromised patients (28.54\%), mentally-ill patients (38.52\%) and other risk groups (35.13\%). Toxoplasma infection among the Mexican population showed a downward trend of $0.1 \% / y e a r$ over a period of sixty years that represents a $5.8 \%$ reduction in prevalence.

Conclusions: This analysis showed a downward trend of infection; however, there are individuals at high risk for infection such as immunocompromised patients, mentally-ill patients and pregnant women. Further research is required to provide better prevention strategies, effective diagnostic testing and medical management of patients. Educational efforts are required to avoid the transmission of infection in populations that cannot be controlled by drugs alone.
\end{abstract}

Keywords: Toxoplasmosis, Toxoplasma infection, Mexican population, Epidemiology, Meta-analysis

\footnotetext{
* Correspondence: mlgalvanr@gmail.com

'Department of Physiology, Neurophysiology Laboratory, Health Sciences University Center, University of Guadalajara, Sierra Mojada \# 950 Edificio N, Col. Independencia, Guadalajara, Jalisco 44320, México

Full list of author information is available at the end of the article
} 


\section{Background}

Toxoplasmosis is a disease caused by Toxoplasma gondii (T. gondii). It was described in a North African rodent (Ctenodactylus gondii) by Nicolle and Manceaux in 1908 [1]. The T. gondii is an obligate intracellular parasite with a complex life cycle, in which homeothermic animals, including humans are capable of acting as intermediate hosts. Humans acquire the parasite by the oral route through the consumption of undercooked meat contaminated with cysts, food products (vegetables and fruits) or water contaminated with oocysts [1,2]. Other routes of transmission are organ transplantation [3,4], blood transfusion [5] and congenital transmission. Butchers, slaughterhouse workers and laboratory personnel that handle cultures and animal models with this parasite are also at risk. However, for the majority of the human population, transmission generally occurs by any of the routes aforementioned [6].

T. gondii is found worldwide because a large variety of animals may harbor the parasite and maintain its dissemination. Its broad geographic location is related to several factors, such as contact with infected cat feces and ingestion of mature oocysts [7], food habits and variations in climate. The later has a significant influence on the habitat of $T$. gondii; for instance, an increase in ambient temperature and precipitation can change the humidity of the soil, so that the sporulated oocysts remain viable in the moist environment for a longer period $[8,9]$.

T. gondii is considered as the most prevalent parasitic zoonotic disease worldwide [10], since at least one-third of the world's population is infected [11]. Infections caused by $T$. gondii are more frequent in temperate zones than in cold ones; thus, France has the highest prevalence of $90 \%$, whereas the lowest prevalence is found in Alaska with only $1 \%$. However, global warming has caused an increase of $T$. gondii infections in different regions of the world as a result of changing environmental conditions [8].

The mean prevalence of $T$. gondii infection among the Mexican general population is $50 \%$; however, there are variations that depend on climate and humidity. Several risk groups have been identified with high prevalence of infection such as cat owners, people who consume raw or undercooked meat, immunocompromised patients and those that undergo organ transplantation [3,4]. Furthermore, we recently carried out a meta-analysis on reports of toxoplasmosis among Mexican newborns. The weighted prevalence in 4833 asymptomatic newborns was $0.616 \%$, whereas, among 895 symptomatic newborns, the weighed prevalence was 3.02\% [12].

Diagnostic testing for toxoplasmosis can be done by staining body fluids or mouse inoculation to see if Toxoplasma parasites develop. Skin test antigen (toxoplasmin)
(STA), and serological procedures such as the Complement Fixation Test (CF), Latex Flocculation Test (LF), Sabin and Feldman (SF), Indirect Haemagglutination Test (IHA), Indirect Immunofluorescence assay (IFI), and Enzyme-linked immunosorbet assay (ELISA) have been employed to detect specific antibodies in screening programs and also as adjuncts to the diagnosis of acute toxoplasmosis. More recently other methods have been developed such as Western-blot and detection of DNA with polymerase chain reaction (PCR) [1,4].

Treatment for human toxoplasmosis is highly important for immunocompromised patients or acutely infected pregnant women. Various pharmacological agents are available such as pyrimethamine alone or combined with sulfadiazine. Atovaquone has been used as a second course of treatment for retinochoroiditis. Azithromycin is used as an alternative in the treatment of ocular and cerebral toxoplasmosis in AIDS patients, as well as, for active, non-vision-threatening toxoplasmic retinochoroiditis with satisfactory results $[1,13,14]$.

The purpose of this systematic review and meta-analysis was to evaluate the seroprevalence of toxoplasmosis and its relationship with different risk factors. The understanding of these relationships can aid in the analysis of the epidemiological pattern of disease among different population groups and the epidemiologic shift of $T$ gondii infection in Mexico.

\section{Methods}

\section{Ethical Aspects}

This study was approved by the Ethical Committee of the Health Sciences Center of the University of Guadalajara \# C.I.100-2012.

\section{Database search}

Five databases were searched (Pub Med, Lilacs, Medline, Latindex and Google Scholar) from January to June of 2012. The following limits were applied: published January 1951 to 2012, the first case of human T. gondii infection was reported in an 11-month old girl from the Pediatric Hospital at Mexico City in 1950 [15], written in English or Spanish and undertaken in adults. The search terms were "infection with Toxoplasma", "toxoplasmosis", "epidemiology", "risk factors", "infection by T. gondii, Mexico" "anti-Toxoplasma antibodies" alone or combined.

\section{Data collection}

All retrieved studies were studied carefully by two investigators (GRML and BR). The extracted data included: year of publication, characteristics of the study population, location of the study, sample size, number of cases, diagnostic test and risk factors. Abstracts were included if considered acceptable, but were not included in the 
meta-analysis for risk factors. Reference lists of full-text publications and textbooks were also examined to identify studies not retrieved by the original search.

\section{Data analysis}

The crude prevalence data and the weighted prevalence were calculated for each study (Table 1) [16-59]. Seven different types of diagnostic tests were identified during the literature search. Table 2 summarizes their main characteristics, sensitivity and specificity, and their timeline of employment in Mexico. Study groups were also stratified according to categories alone (Table 3) or by combining the risk group and diagnostic test used in each study [60-66] (Table 4).

The study groups were divided into: 1) Individuals with risk factors were designated as high risk groups that included: women who had had abortions, immunocompromised patients with AIDS or HIV, leprous people, patients with neurological disorders, pet-cat, owners and slaughterhouse workers; 2) Individuals without risks factors were designated in low risk groups: blood donors, general population and normal pregnant women.

\section{Statistical methods Crude prevalence}

The crude prevalence of each study group was estimated to assess the amount of affection by the disease expressed in percentage of positives cases in relation to all cases analyzed. It was calculated as the number of positive cases divided by the sample size of the cohort in each study group.

\section{Weighted population prevalence (WP)}

The assessment of the prevalence of $T$. gondii of the different study groups was estimated by using the weighed population prevalence, given that not all the studies included the same number of individuals. This strategy restricts the bias that may impose the heterogeneous nature of the reports, and has proven to be valid when combining a number of studies with inherent heterogeneity in sample size and effects [67].

Each crude prevalence was multiplied by a "weight", which was proportional to the number of subjects included in the sample, i.e., in large samples, the prevalence found outweighs that of small samples. This prevalence is obtained by summing the product of the prevalence for its "weight" of the sum of the "weights". This estimate is more accurate than the overall crude prevalence to estimate the true prevalence of a cumulative set of groups. The formula to calculate the population prevalence (weighed prevalence, WP) of $T$. gondii in all groups or subgroups, included in this meta-analysis was $\mathrm{P}=\sum(\mathrm{pi})(1 / \mathrm{vi}) / \sum 1 / \mathrm{vi}$ as explained by Borenstein et al. [68].

\section{Definitions}

$\mathrm{i}=$ Number of studies in each group. $\mathrm{Ni}=$ Total number of cases in each study, $\mathrm{Ai}=$ Number of positive cases from each study, (Ni-Ai) is the number of negative cases in each study. The risk of infection as a proportion in each study (pi) was calculated as $\mathrm{Ai} / \mathrm{Ni}$. The variance of each study (vi) was calculated as $\mathrm{Ai}(\mathrm{Ni}-\mathrm{Ai}) / \mathrm{Ni}^{3}$. The standard error (SEi) of each study was estimated as $\sqrt{ }$ vi.

The total population variance $(\mathrm{V})$ was estimated as $1 / \sum 1 /$ vi. The standard error of the population was calculated as $\mathrm{SE}=\sqrt{\mathrm{V}}$. The confidence interval (C.I.95\%) for the population prevalence was obtained by $\mathrm{P}+1.96$ SE (upper limit) and P-1.96 EE lower limit. The probability that the prevalence could be different from zero was calculated with a $\mathrm{Z}$ test, $\mathrm{Z}=\mathrm{P} / \mathrm{SE}$.

\section{Forest plot}

A Forest Plot chart was built in order to provide a comprehensive analysis of the studies included in the meta-analysis according to its odd ratio and confidence interval (CI) [68].

\section{Linear regression analysis}

A bivariate linear regression analysis was conducted to determine the relationship between the seroprevalence of Toxoplasma infection over time. The regression coefficient was calculated by the equation $(y=a+b x)$, $\mathrm{a}=$ ordinate of origin, $\mathrm{b}=$ slope and the $\mathrm{R}^{2}$ and $\mathrm{p}$ were obtained with the SPSS program (Version 18). The epidemiological behaviour of the prevalence of Toxoplasma infection was estimated by plotting the year of each publication date (independent variable) starting at year 1951 until 2012 versus the relative prevalence (dependent variable) reported in each study [68].

\section{Results}

From the five databases, a total of 45 publications were eligible that included 132 studies and 70,123 individuals, and 19,262 positive cases as shown in Table 1. As shown in Table 3, most of the studies were carried out in low risk groups, such as, the general population $(n=90$ studies) followed by pregnant women $(n=12)$, with the least number of studies in blood donors $(n=4)$. In the high-risk groups, relatively fewer studies have been carried out; women with miscarriage $(n=3)$, immunocompromised patients $(n=7)$, mentally-ill patients $(n=4)$, other risk groups $(n=5)$ and patients with nonrelated comorbidity $(n=7)$. To the best of our knowledge, all the studies included in this meta-analysis had a crosssectional design and were aimed to identify the prevalence of $T$. gondii antibodies in a cohort. No prospective or follow-up studies aimed to seek seroconversion or self-reported results were detected. 
Table 1 Publications included for meta-analysis with diagnostic methods and population characteristics

\begin{tabular}{|c|c|c|c|c|c|c|c|c|c|c|}
\hline Sequence & Year & First Author & State & Municipality & Test & Category & $\begin{array}{c}\text { Number } \\
\text { of } \\
\text { cases }\end{array}$ & $\begin{array}{c}\text { Positive } \\
\text { cases }\end{array}$ & $\begin{array}{c}\text { Prevalence } \\
\text { (\%) }\end{array}$ & Reference \\
\hline 1 & 1951 & BiagiF. & Tamaulipas & Tampico & STA & GP & 231 & 108 & 47 & {$[16]$} \\
\hline 2 & 1952 & Biagi $F$. & México & Mexico & STA & IC & 155 & 58 & 37.4 & [49] \\
\hline 3 & 1952 & Bustos C. & Veracruz & Orizaba & STA & GP & 86 & 31 & 51 & {$[17]$} \\
\hline 4 & 1953 & Biagi F. & Campeche & Escarcega & STA & GP & 132 & 76 & 56.8 & [18] \\
\hline 5 & 1953 & Varela G. & $\begin{array}{l}\text { Estado de } \\
\text { Mexico }\end{array}$ & Toluca & STA & GP & 500 & 81 & 16 & [19] \\
\hline 6 & & Varela G. & Mexico & México & STA & $C M$ & 116 & 19 & 16 & [19] \\
\hline 7 & & Varela G. & Mexico & México & STA & $\mathrm{CM}$ & 47 & 7 & 14.8 & [19] \\
\hline 8 & & Varela G. & Mexico & México & STA & $C M$ & 102 & 13 & 12.7 & [19] \\
\hline 9 & & Varela G. & & Zoquiapan & STA & IC & 107 & 13 & 14 & [19] \\
\hline 10 & 1954 & Gutierrez E.B. & México & México & CFT & $\mathrm{Ml}$ & 58 & 14 & 24.14 & [53] \\
\hline 11 & 1955 & Varela G. & Mexico & México & SF & GP & 60 & 16 & 26.7 & [20] \\
\hline 12 & & Varela G. & Michoacan & N.E. & SF & GP & 22 & 6 & 27.3 & [20] \\
\hline 13 & & Varela G. & Mexico & México & SF & GP & 104 & 39 & 37.5 & {$[20]$} \\
\hline 14 & & Varela G. & Oaxaca & Oaxaca & SF & GP & 276 & 96 & 34.8 & {$[20]$} \\
\hline 15 & & Varela G. & Tamaulipas & N.E. & SF & GP & 230 & 90 & 39 & [20] \\
\hline 16 & & Varela G. & Yucatan & N.E. & SF & GP & 17 & 11 & 64.7 & [20] \\
\hline 17 & & Varela G. & $\begin{array}{l}\text { Distrito } \\
\text { federal }\end{array}$ & Mexico & SF & $\mathrm{Ml}$ & 91 & 54 & 59 & [20] \\
\hline 18 & & Varela G. & Puebla & N.E. & SF & GP & 44 & 41 & 93.2 & [20] \\
\hline 19 & 1957 & Biagi $F$. & México & Ixtapalapa, D.F. & STA & GP & 272 & 37 & 13.6 & [21] \\
\hline 20 & 1961 & Varela G. & Baja, California & Mexicali & SF & GP & 73 & 26 & 35.9 & [22] \\
\hline 21 & & Varela G. & Chihuahua & Chihuahua & SF & GP & 12 & 3 & 25 & {$[22]$} \\
\hline 22 & & Varela G. & México & Distrito Federal & SF & GP & 2,463 & 783 & 31.8 & [22] \\
\hline 23 & & Varela G. & Hidalgo & Apan & SF & GP & 409 & 117 & 28.6 & [22] \\
\hline 24 & & Varela G. & $\begin{array}{l}\text { Estado de } \\
\text { Mexico }\end{array}$ & Toluca & SF & GP & 64 & 15 & 23.4 & [22] \\
\hline 25 & & Varela G. & Michoacan & Morelia & SF & GP & 35 & 11 & 31.42 & {$[22]$} \\
\hline 26 & & Varela G. & Morelos & Various & SF & GP & 208 & 54 & 25.96 & {$[22]$} \\
\hline 27 & & Varela G. & Nayarit & Tepic & SF & GP & 112 & 25 & 22.3 & [22] \\
\hline 28 & & Varela G. & Oaxaca & $\begin{array}{l}\text { Oaxaca and } \\
\text { Tuxtepec }\end{array}$ & SF & GP & 546 & 107 & 19.59 & [22] \\
\hline 29 & & Varela G. & Puebla & Puebla & SF & GP & 170 & 61 & 35.9 & {$[22]$} \\
\hline 30 & & Varela G. & Queretaro & Various & SF & GP & 90 & 33 & 36.66 & [22] \\
\hline 31 & & Varela G. & Sinaloa & Culiacan & SF & GP & 311 & 109 & 35 & [22] \\
\hline 32 & & Varela G. & Sinaloa & Mazatlan & SF & GP & 100 & 16 & 16 & {$[22]$} \\
\hline 33 & & Varela G. & Tabasco & Macuspana & SF & GP & 108 & 38 & 35.2 & [22] \\
\hline 34 & & Varela G. & Tamaulipas & Ciudad Victoria & SF & GP & 220 & 39 & 17.7 & [22] \\
\hline 35 & & Varela G. & Tamaulipas & Nuevo Laredo & SF & GP & 100 & 28 & 28 & [22] \\
\hline 36 & & Varela G. & Tlaxcala & Tlaxcala & SF & GP & 594 & 144 & 24.2 & {$[22]$} \\
\hline 37 & & Varela G. & Veracruz & Boca del Rio & SF & GP & 93 & 36 & 38.7 & [22] \\
\hline 38 & & Varela G. & Veracruz & Soconusco & SF & GP & 121 & 39 & 32.2 & [22] \\
\hline 39 & & Varela G. & Veracruz & Veracruz & SF & GP & 125 & 30 & 24 & {$[22]$} \\
\hline 40 & & Varela G. & Yucatan & Merida & SF & GP & 17 & 11 & 64.7 & [22] \\
\hline
\end{tabular}


Table 1 Publications included for meta-analysis with diagnostic methods and population characteristics (Continued)

\begin{tabular}{|c|c|c|c|c|c|c|c|c|c|c|}
\hline 41 & 1962 & Carrillo C. & México & Mexico & SF & $\mathrm{BD}$ & 232 & 73 & 31.4 & [46] \\
\hline 42 & 1965 & Espinosa de los Reyes VM, & México & Mexico & SF & PW & 329 & 112 & 34 & [37] \\
\hline 43 & 1966 & Roch E. & $\begin{array}{l}\text { Distrito } \\
\text { Federal }\end{array}$ & México & SF & MW & 2,320 & 815 & 35.13 & [44] \\
\hline 44 & 1966 & Roch E. & Mexico & All States & SF & GP & 14,869 & 4,411 & 30 & [23] \\
\hline 45 & 1972 & Goldsmith RS. & Oaxaca & Puerto Escondido & $\mathrm{IHA}$ & GP & 159 & 2 & 1.26 & [24] \\
\hline 46 & 1972 & Goldsmith RS. & Oaxaca & Mixteca Alta & $\mathrm{IHA}$ & GP & 114 & 0 & 0 & [24] \\
\hline 47 & 1972 & Goldsmith RS. & Oaxaca & Ixtlan & $\mathrm{IHA}$ & GP & 48 & 2 & 4.2 & [24] \\
\hline 48 & 1972 & Goldsmith RS. & Oaxaca & Región del Valle & $\mathrm{IHA}$ & GP & 150 & 5 & 3.3 & [24] \\
\hline 49 & 1972 & Goldsmith RS. & Oaxaca & Tehuantepec & $\mathrm{IHA}$ & GP & 137 & 18 & 13 & [24] \\
\hline 50 & 1974 & Biagi F. & Mexico & México & $\mathrm{FL}$ & WP & 367 & 73 & 19.9 & [35] \\
\hline 51 & 1986 & Fernandez Terrano & Tabasco & $\begin{array}{l}\text { Region de los } \\
\text { Rios }\end{array}$ & $\mathrm{IFI}-\mathrm{G}$ & WP & 125 & 75 & 60 & [36] \\
\hline 52 & 1989 & Galvan-Ramirez ML. & & Jalisco & $\mathrm{IFI}-\mathrm{G}$ & GP & 807 & 25 & 3.1 & [25] \\
\hline 53 & 1989 & Zavala-Velazquez J. & Yucatan & Merida & $\mathrm{IFI}-\mathrm{G}$ & MW & 100 & 47 & 47 & [45] \\
\hline 54 & 1991 & Goldsmith RS. & Oaxaca & 60 municipalities & $\mathrm{IHA}$ & GP & 3,229 & 124 & 3.8 & [26] \\
\hline $55-86$ & 1991 & Velasco- Castrejon O. & Mexico & All states & $\mathrm{IFI}-\mathrm{G}$ & GP & 29,279 & 9,371 & 32 & [27] \\
\hline 87 & 1995 & Galvan-Ramirez ML. & Jalisco & Guadalajara & ELISA-G & PW & 350 & 122 & 34.9 & [39] \\
\hline 88 & & Galvan-Ramirez ML & Jalisco & Guadalajara & ELISA-G & MW & 105 & 48 & 44.9 & [39] \\
\hline 89 & & Galvan-Ramirez ML & Jalisco & Guadalajara & ELISA-G & PW & 50 & 13 & 26.01 & [39] \\
\hline 90 & 1997 & Galvan-Ramirez ML & Jalisco & Guadalajara & ELISA-G & IC & 39 & 27 & 69.2 & [50] \\
\hline 91 & & Galvan-Ramirez ML & Jalisco & Guadalajara & ELISA-G & IC & 53 & 19 & 35.8 & [50] \\
\hline 92 & 1997 & Tay J. & Distrito Fed. & México & ELISA-G & $\mathrm{Ml}$ & 328 & 125 & 38 & [54] \\
\hline 93 & 1998 & Gongora R. & Yucatan & Mérida & ELISA-G & IC & 95 & 45 & 47 & [47] \\
\hline 95 & & Gongora R. & Yucatan & Mérida & ELISA-G & $\mathrm{BD}$ & 100 & 69 & 69 & [47] \\
\hline 93 & 1999 & Galvan-Ramirez ML & Jalisco & Guadalajara & ELISA-G & ORG & 59 & 38 & 64 & [7] \\
\hline 96 & 2000 & Kelso Santos E. & Nuevo Leon & Monterrey & ELISA-G & GP & 400 & 82 & 20.5 & [29] \\
\hline 97 & 2003 & Jaramillo P.J. & $\begin{array}{l}\text { Estado de } \\
\text { Mexico }\end{array}$ & Toluca & ELISA-G & PW & 372 & 47 & 12.61 & [40] \\
\hline 98 & 2004 & Galvan-Ramirez ML & Jalisco & Guadalajara & ELISA-G & PW & 30 & 14 & 47 & [38] \\
\hline 99 & & Galvan-Ramirez ML & Jalisco & Guadalajara & ELISA-G & PW & 30 & 13 & 43 & [38] \\
\hline 100 & & Galvan-Ramirez ML & Jalisco & Guadalajara & ELISA-G & PW & 60 & 17 & 28.3 & [38] \\
\hline 101 & 2005 & Galvan-Ramirez ML & Jalisco & Guadalajara & ELISA-G & $\mathrm{BD}$ & 359 & 104 & 29 & {$[5]$} \\
\hline 102 & 2006 & Alvarado-Esquivel C. & Durago & Guadalajara & ELISA-G & PW & 343 & 21 & 6.1 & [41] \\
\hline 103 & 2007 & Alvarado-Esquivel C. & Durango & Durango & ELISA-G & $\mathrm{BD}$ & 432 & 32 & 7.4 & [48] \\
\hline 104 & 2008 & Alvarado-Esquivel C. & Durango & Durango & ELISA-G & ORG & 90 & 19 & 21.1 & [58] \\
\hline 105 & & Alvarado-Esquivel C. & Durango & Durango & ELISA-G & ORG & 83 & 7 & 8.4 & [58] \\
\hline 106 & 2008 & Alvarado-Esquivel C. & Durango & Durango & ELISA-G & GP & 187 & 67 & 35.8 & [30] \\
\hline 107 & 2008 & Alvarado-Esquivel C. & Durango & Durango & ELISA-G & GP & 121 & 20 & 16.5 & [30] \\
\hline 108 & 2008 & Alvarado-Esquivel C. & Durango & Durango & ELISA-G & GP & 155 & 23 & 14.8 & [30] \\
\hline 109 & 2008 & Galvan-Ramirez ML. & Jalisco & Guadalajara & ELISA-G & ORG & 145 & 104 & 72 & {$[6]$} \\
\hline 110 & 2009 & Alvarado-Esquivel C. & Durango & Guadalajara & ELISA-G & $P G$ & 439 & 36 & 8.2 & {$[42]$} \\
\hline 111 & 2009 & Cañedo-Solares I. & $\begin{array}{l}\text { Distrito } \\
\text { Federal }\end{array}$ & Mexico & ELISA-G & $P G$ & 100 & 30 & 30 & [43] \\
\hline 112 & 2010 & Alvarado-Esquivel C. & Durango & Durango & ELISA-G & GP & 248 & 22 & 8 & [31] \\
\hline 113 & 2010 & Alvarado-Esquivel C. & Durango & Durango & ELISA-G & GP & 61 & 4 & 6.6 & {$[56]$} \\
\hline
\end{tabular}


Table 1 Publications included for meta-analysis with diagnostic methods and population characteristics (Continued)

\begin{tabular}{|c|c|c|c|c|c|c|c|c|c|c|}
\hline 114 & & Alvarado-Esquivel C. & Durango & Durango & ELISA-G & GP & 203 & 17 & 8.4 & {$[56]$} \\
\hline 115 & & Alvarado-Esquivel C. & Durango & Durango & ELISA-G & GP & 168 & 10 & 6.6 & [56] \\
\hline 116 & 2010 & Alvarado-Esquivel C. & Durago & Durango & ELISA-G & GP & 152 & 46 & 30 & [31] \\
\hline 117 & 2010 & Galvan-Ramirez ML & Jalisco & Guadalajara & ELISA-G & GP & 174 & 30 & 17.8 & [28] \\
\hline 118 & 2010 & Alvarado-Esquivel C. & Durango & Durango & ELISA-G & CM & 85 & 7 & 8.2 & [51] \\
\hline 119 & & Alvarado-Esquivel C. & Durango & Durango & ELISA-G & CM & 50 & 5 & 10 & [51] \\
\hline 120 & & Alvarado-Esquivel C. & Durango & Durango & ELISA-G & $\mathrm{CM}$ & 234 & 28 & 12 & [51] \\
\hline 121 & & Alvarado-Esquivel C. & Durango & Durango & ELISA-G & IC & 103 & 7 & 6.8 & [51] \\
\hline 122 & 2011 & Alvarado-Esquivel C. & Durango & Durango & ELISA-G & ORG & 124 & 8 & 7 & {$[57]$} \\
\hline 123 & 2011 & Alvarado-Esquivel C. & Durango & Durango & ELISA-G & Ml & 50 & 10 & 20 & [55] \\
\hline 124 & 2011 & Alvarado-Esquivel C. & Durango & Durango & ELISA-G & GP & 150 & 8 & 5.3 & [32] \\
\hline 125 & 2011 & Alvarado-Esquivel C. & Durango & Durango & ELISA-G & IC & 75 & 10 & 13.3 & {$[52]$} \\
\hline 126 & & Alvarado-Esquivel C. & Durango & Durango & ELISA-G & GP & 150 & 16 & 10.7 & {$[52]$} \\
\hline 127 & 2011 & Alvarado-Esquivel C. & Durango & Durango & ELISA-G & GP & 1,101 & 76 & 6.9 & [59] \\
\hline 128 & & Alvarado-Esquivel C. & Durango & Durango & ELISA-G & CM & 55 & 9 & 16.4 & [59] \\
\hline 129 & 2011 & Alvarado-Esquivel C. & Durango & Durango & ELISA-G & GP & 974 & 59 & 6.1 & [32] \\
\hline 130 & 2012 & Alvarado-Esquivel C. & Durango & Durango & ELISA-G & GP & 133 & 11 & 8.3 & [33] \\
\hline 131 & & Alvarado-Esquivel C. & Durango & Durango & ELISA-G & GP & 266 & 14 & 5.3 & [33] \\
\hline 132 & 2012 & Alvarado-Esquivel C. & Durango & Durango & ELISA-G & GP & 156 & 35 & 22.4 & [34] \\
\hline Total & & & & & & & 70,123 & 19,262 & & \\
\hline
\end{tabular}

Skin test antigen (toxoplasmin) (STA); Complement Fixation Test (CF); Latex Flocculation Test (LF); Sabin \& Feldman (SF); Indirect Haemagglutination Test (IHA); Enzyme-linked immunosorbet assay and type of antibody detected (ELISA-G), Indirect Immunofluorescence assay and type of antibody detected (IFI-G); General Population (GP); Pregnant Women (PW); Blood Donors (BD); Patients with Comorbidity (CM); Immunocompromised patients (IC); Women with Miscarriages (MW); Mentally-ill patients (Ml); Other risk groups (ORG).

Table 2 Diagnostic methods

\begin{tabular}{|c|c|c|c|c|c|}
\hline Diagnostic test & Fundament & Sensitivity & Specificity & Timeline & References \\
\hline Skin test antigen (STA & $\begin{array}{l}\text { Type IV cell-mediated hypersensitivity } \\
\text { reaction against the T.gondii antigen. }\end{array}$ & $80 \%$ & $70 \%$ & 1950-1951 & {$[16-19,21,49]$} \\
\hline $\begin{array}{l}\text { Sabin and Feldman } \\
\text { Dye Test (SF). }\end{array}$ & $\begin{array}{l}\text { The gold standard. A dye test in which the } \\
\text { serum antibodies alter the staining pattern } \\
\text { of the T. gondii tachyzoites. }\end{array}$ & $96 \%$ & $98 \%$ & $1955-2005$ & {$[20,22,23,37,44,46]$} \\
\hline $\begin{array}{l}\text { Complement Fixation } \\
\text { Test (CF). }\end{array}$ & $\begin{array}{l}\text { Antigen-antibody complexes are formed } \\
\text { and detected by using a standard system } \\
\text { with hemolisin and complement }\end{array}$ & $97.1 \%$ & $64.5 \%$ & 1954-1982 & [53] \\
\hline $\begin{array}{l}\text { Látex Flocculation } \\
\text { Test (LF). }\end{array}$ & $\begin{array}{l}\text { This test uses latex particles for } \\
\text { antigen-antibody flocculation. }\end{array}$ & No reported. & & 1974 & {$[35]$} \\
\hline $\begin{array}{l}\text { Indirect Fluorescent } \\
\text { Antibody Test (IFI). }\end{array}$ & $\begin{array}{l}\text { Tachyzoites are fixed on a slide and } \\
\text { exposed to test serum, then washed } \\
\text { and exposed to a standard antibody } \\
\text { labeled with fluorescent dye. }\end{array}$ & $95 \%$ & $96 \%$ & 1986 to date & {$[5,25,27,28,36,38,54]$} \\
\hline $\begin{array}{l}\text { Indirect } \\
\text { Haemagglutination } \\
\text { Test (IHA). }\end{array}$ & $\begin{array}{l}\text { This test uses sheep red cells exposed } \\
\text { to tannic acid and then to the soluble } \\
\text { antigen fixed at } 37^{\circ} \mathrm{C} \text {. }\end{array}$ & $95 \%$ & $96 \%$ & 1972 to 1989 & {$[24,26]$} \\
\hline $\begin{array}{l}\text { Enzyme-linked } \\
\text { immunoabsorbent } \\
\text { assay (ELISA). }\end{array}$ & $\begin{array}{l}\text { The ELISA detects T. gondii immunoglobulin } \\
\text { IgG e IgM in serum and other body fluids } \\
\text { with antibodies marked with peroxidase } \\
\text { and fosfatase enzymes }\end{array}$ & $100 \%$ & $98.4 \%$ & 1995 to date & {$[6,7,29-34,39-43,47,48,50-52,55,56]$} \\
\hline
\end{tabular}


Table 3 Crude and weighted Toxoplasma infection prevalence in low and high risk groups of Mexican population

\begin{tabular}{|c|c|c|c|c|c|c|c|}
\hline Population studied & $\begin{array}{c}\text { Number } \\
\text { of } \\
\text { studies }\end{array}$ & $\begin{array}{c}\text { Number } \\
\text { of } \\
\text { cases }\end{array}$ & $\begin{array}{l}\text { Positive } \\
\text { cases }\end{array}$ & $\begin{array}{l}\text { A/Nx100 Crude } \\
\text { prevalence (\%) }\end{array}$ & $\begin{array}{c}\text { Weighed } \\
\text { prevalence (\%) }\end{array}$ & $\begin{array}{c}\text { CI 95\% } \\
\text { Lower-upper } \\
\text { limit (\%) }\end{array}$ & References \\
\hline \multicolumn{8}{|l|}{ Low Risk Groups } \\
\hline General population & 90 & 61536 & 16855 & 27.39 & 20.26 & $18.78-19.36$ & {$[16-24,34,51,52,55,56,58,59]$} \\
\hline Pregnant Women & 12 & 2595 & 573 & 22.08 & 15.62 & $14.30-16.93$ & {$[35-43]$} \\
\hline Blood Donors & 4 & 1123 & 278 & 24.76 & 17.035 & $15.03-19.03$ & {$[5,46-48]$} \\
\hline Patients with comorbidity & 7 & 689 & 88 & 12.77 & 12.27 & $9.83-14.72$ & {$[19,51,59]$} \\
\hline \multicolumn{8}{|l|}{ High Risk Groups } \\
\hline Women with miscarriages & 3 & 2525 & 910 & 36.03 & 35.96 & $34.1-37.83$ & {$[39,44,45]$} \\
\hline Immunocompromised patients & 7 & 627 & 179 & 28.54 & 20.2 & $17.35-23.05$ & {$[19,47,49-52]$} \\
\hline Mentally-ill patients & 4 & 527 & 203 & 38.52 & 37.24 & $33.24-41.26$ & {$[20,53-55]$} \\
\hline Other risk groups & 5 & 501 & 176 & 35.13 & 21.88 & $19.0-24.76$ & {$[6,7,57,58]$} \\
\hline Total & 132 & 70,123 & 19,262 & & & & \\
\hline
\end{tabular}

Meta-analysis in low risk groups

\section{General population}

Most of the studies were carried out in low risk groups such as the general population (90 studies) that gave a total of 61,536 people tested for $T$. gondii infection. The WP was $20.26 \%\left(\mathrm{CI}_{95 \%} 18.78 \%-19.36 \%\right)$ with a variance of $0.0002 \%$ and a standard error of $0.1463 \%, Z=130.391$ and $\mathrm{p}<0.001$ (Table 3).

\section{Pregnant women}

In this study group, 12 publications included 2,595 pregnant women. The WP was $15.62 \% \quad\left(\mathrm{CI}_{95 \%} 14.30 \%\right.$ $16.93 \%)$, with a variance of $0.0045 \%$ and standard error of $0.67 \%, \mathrm{Z}=23.313$ and $\mathrm{p}<0.001$ (Table 3 ).

\section{Blood donors}

In 4 studies, a total of 1123 blood donors were tested. The WP was $17.03 \%\left(\mathrm{CI}_{95 \%} 15.03 \%\right.$ - 19.03\%) with a variance of $0.00104 \%$ and standard error of $1.0213 \%$, $\mathrm{Z}=16.679$ and $\mathrm{p}<0.001$ (Table 3).

\section{Patients with comorbidity}

In 689 cases from seven studies, a WP of $12.27 \%$ $\left(\mathrm{CI}_{95 \%} 9.83-14.72\right)$ was found with a variance of $0.0155 \%$, standard error of $1.2463 \%, Z=9.85$ and $p<0.001$ (Table 3).

\section{Meta-analysis in high-risk groups \\ Women with miscarriages}

In 3 studies, 2595 women were tested. The WP was $35.96 \%\left(\mathrm{CI}_{95 \%} 34.10 \%\right.$ - 37.83\%) with a variance of $0.0091 \%$ and standard error of $0.9532 \%, Z=37.73$ and p $<0.001$ (Table 3).

\section{Immunocompromised patients}

In this study group, 627 patients from seven studies were included. The WP was $20.20 \%\left(\mathrm{CI}_{95 \%} 17.35 \%-23.05 \%\right)$

Table 4 Weighted prevalence of $T$. gondii infection adjusted by risk factor (with or without) and diagnostic method

\begin{tabular}{|c|c|c|c|c|c|c|c|}
\hline $\begin{array}{l}\text { Population studied-diagnostic } \\
\text { method }\end{array}$ & $\begin{array}{l}\text { Number } \\
\text { of groups }\end{array}$ & $\begin{array}{l}\text { Number } \\
\text { of cases }\end{array}$ & $\begin{array}{l}\text { Positive } \\
\text { cases }\end{array}$ & $\begin{array}{c}\mathrm{A} / \mathrm{Nx} 100 \\
\text { prevalence (\%) }\end{array}$ & $\begin{array}{l}\text { Meta-analysis } \\
\text { prevalence (\%) }\end{array}$ & $\begin{array}{l}\text { Lower } \\
\text { limit (\%) }\end{array}$ & $\begin{array}{l}\text { Upper } \\
\text { limit (\%) }\end{array}$ \\
\hline with Risk - STA & 4 & 480 & 103 & 21.45 & 13.39 & 10.35 & 16.44 \\
\hline without Risk -STA & 6 & 1268 & 340 & 22.81 & 22.48 & 20.32 & 24.65 \\
\hline without Risk-LF & 1 & 367 & 73 & 19.89 & 19.89 & 15.8 & 23.97 \\
\hline without-risk CF & 1 & 58 & 14 & 24.14 & 22.16 & 13.64 & 35.15 \\
\hline without Risk-SF & 32 & 22245 & 6674 & 30 & 30 & 29.4 & 30.59 \\
\hline with Risk-SF & 1 & 2320 & 815 & 35.13 & 35.12 & 33.18 & 37.18 \\
\hline without Risk-IFI-G & 36 & 29997 & 9597 & 31.99 & 29.3 & 28.81 & 29.79 \\
\hline with Risk-IFI-G & 5 & 1295 & 224 & 16.77 & 5.58 & 4.44 & 6.77 \\
\hline without Risk-IHA & 6 & 3837 & 151 & 3.94 & 2.63 & 1.24 & 4.02 \\
\hline with Risk-ELISA-G & 18 & 1963 & 523 & 26.64 & 17.66 & 16.17 & 19.15 \\
\hline without Risk-ELISA-G & 22 & 6293 & 748 & 11.89 & 8.92 & 8.24 & 9.6 \\
\hline Total & 132 & 70,123 & 19,262 & & & & \\
\hline
\end{tabular}

Complement Fixation (CF), Skin test antigen toxoplasmin (STA), Latex Floculation (LF), Sabin and Feldman (SF), Indirect Hemaglutination (IHA), Indirect Fluorescence (IFI), Enzyme-linked immunosorbet assay (ELISA). All prevalences calculated by meta-analysis were statistically significant ( $<<0.001)$. 
with a variance of $0.0211 \%$ and standard error of $1.45 \%$, $\mathrm{Z}=13.90$ and $\mathrm{p}<0.0001$ (Table 3).

\section{Mentally-ill patients}

A total of 527 mentally-ill patients were included from four studies. The WP was $37.24 \%\left(\mathrm{CI}_{95 \%} 33.24 \%-41.26 \%\right)$ with a variance of $0.0994 \%$ and standard error of $3.15 \%$, $\mathrm{Z}=11.43$ and $\mathrm{p}<0.001$ (Table 3).

\section{Other risk groups}

In 501 cases from five studies, a WP of $21.88 \%$ $\left(\mathrm{CI}_{95 \%} 19.00 \%-24.76 \%\right)$ was found with a variance of $0.0215 \%$, standard error of $1.46 \%, Z=14.91$ and $p<0.001$ (Table 3).

\section{Weighted prevalence by diagnostic test and risk factors}

In order to determine, if the prevalence of T. gondii was based on the diagnostic test, the study groups were adjusted by diagnostic test and risk factors reported. Only the studies that used the SF assay and ELISA showed a correlation between the low and high risk. Interestingly, the rest of the diagnostic tests showed a lower prevalence, although it is noteworthy to mention that the difference may be due to the number of cases in the risk group categories (Table 4).

\section{Epidemiological transition of Toxoplasma infection in Mexico}

Figure 1 illustrates the Forest Plot analysis of the WP $\left(\mathrm{CI}_{95 \%}\right)$ of each study included in this meta-analysis. The linear regression analysis of Toxoplasma infection in the Mexican population over a time span of 60 years showed a downward trend of $0.1 \% /$ year that represents an overall $5.8 \%$ reduction of infection $\left(R^{2}=0.0354\right.$ and $F$ $(1)=4.802, p>0.05)$ that was not statistically significant (Figure 2).

\section{Discussion}

The questions that led us to achieve this study were if Mexico had a significant prevalence of toxoplasmosis, and if there have been changes in the epidemiology of this disease from 1951 to date. Five databases were searched, and 132 studies were selected involving 70,123 individuals and 19,262 positive cases.

This study showed that the WP of toxoplasmosis in Mexico was $20.26 \%$, which is relatively low compared to the mean prevalence of $32 \%$ reported in 1991 [27]. This can be explained if we note that variations between methodologies can result in heterogeneity of the estimations. However, despite this situation, at least $20.26 \%$ of Mexicans may have been exposed to toxoplasmosis.

Between 1951 and 1958, the STA was an appropriate test since, at that time, it was a rapid and sensitive assay for epidemiological studies. It did not give cross-reactions with other parasites, but it did make a fair amount of false-negatives in mild infections. This was one of its disadvantages when used for a survey in the general population in Tamaulipas, Campeche and Mexico City (1953). The WP was $21.31 \%$ in 11 study groups with 1846 cases [16-20]. Since only one study was performed by the FL test with 367 individuals, and another study included 58 cases tested by fixation complement, we were not able to compare these results against the SF or IFI assays.

The SF test was used in epidemiological studies carried out between 1961 and 1966. The CP and WP was 30\% and $35.12 \%$, respectively [23]. The advantage of this test

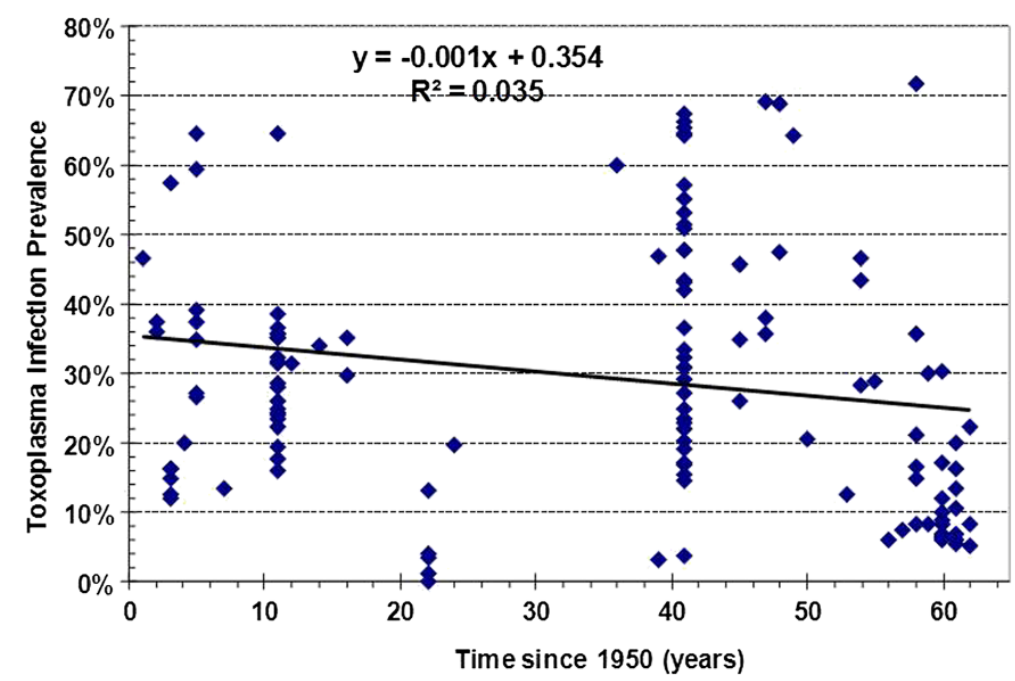

Figure 1 Epidemiological transition of the $T$. gondii infection from 1954 to 2012, (-) Linear regression and triangle ( $\$$ ) is the individual prevalence of Toxoplasma gondii infection reported in each study group. The decrement in the prevalence rate was $0.1 \% / y e a r$. The $\mathrm{R}^{2}$ value was not statistically significant (NS). 


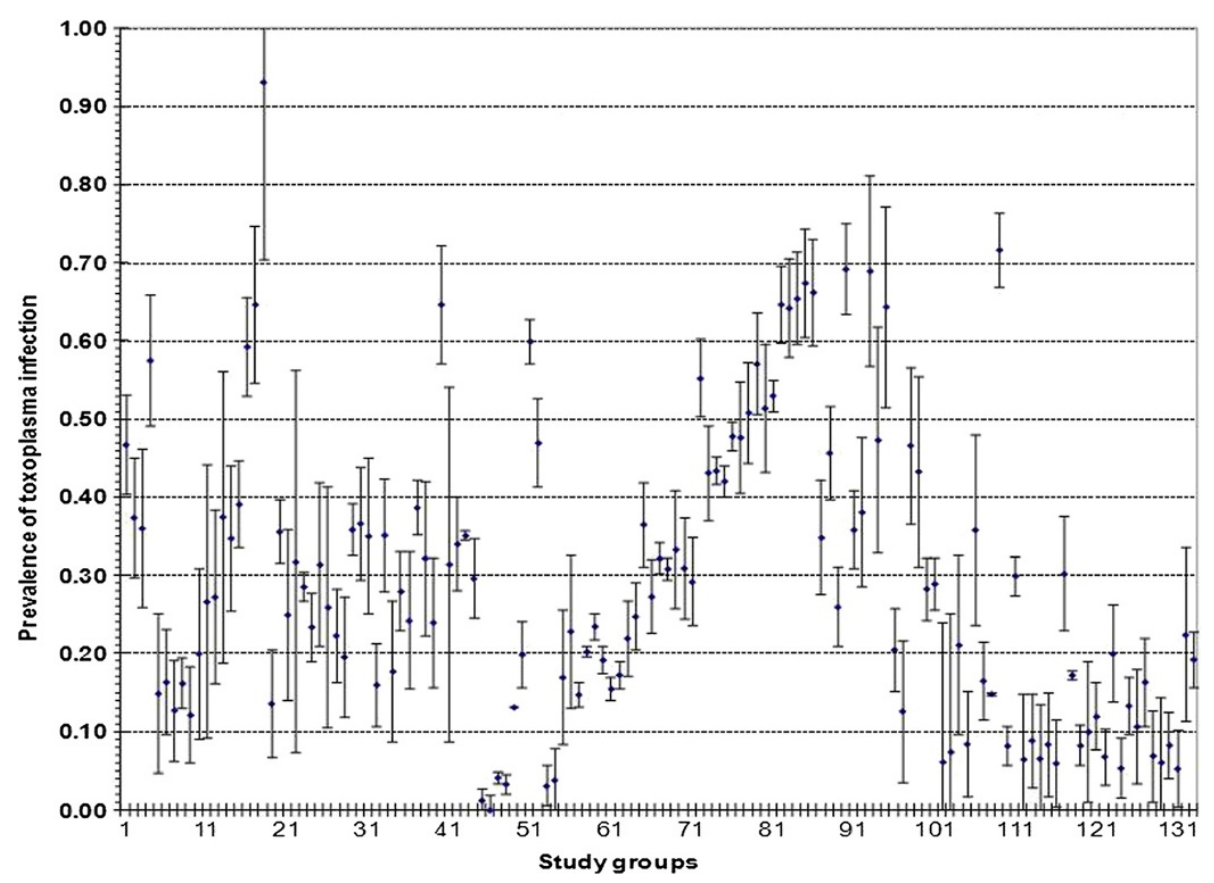

Figure 2 Weighted prevalence of Toxoplasma gondii infection from 132 studies. Forest plot shows event numbers, total numbers and confidence intervals for all study groups included in the meta-analysis. The triangle $(\downarrow)$ represents the prevalence of each study and (T $\perp)$ are intervals of confidence.

is that it is a gold standard, and it is highly sensitive, although the disadvantage is that it requires live parasites. The global prevalence during that time was $14.92 \%$, which is closer, to the prevalence of $8.26 \%$ aforementioned. In the same period, the IFI test was introduced and was then used in the largest survey carried out in Mexico (1992) with 29, 279 people with a mean prevalence of $32.0 \%$ [27]. The meta-analysis of all tests performed with IFI was $29.32 \%$, because the majority of studies have used this method (31,412 cases).

In the 80s, the ELISA methods were introduced with higher sensitivity and specificity that reduced the number of cross-reactions. Interestingly, the WP was lower, ranging from $8.92 \%$ to $17.66 \%$, which may have been caused by the lower number of cases, (8256 cases), less than one-third of the studies, when compared to the 26,751 cases tested with SF and the 30,485 cases with IFI.

The general population involved 61,536 cases with a WP of $20.26 \%$, while the mean prevalence was reported as $32 \%$ in 1991 [27]. This discrepancy may be caused by the differences in the diagnostic methods that are then adjusted when the weighted prevalence was estimated [27].

Pregnant women presented a WP of $15.62 \%$ with an upper limit of $16.93 \%$ that is closer to the general population prevalence (20.26\%), which is reasonable since these women did not have obstetric complications. These values were lower compared to $45.8 \%$ and $30.5 \%$, respectively for specific IgG antibodies detected by the Sabin-Feldman dye test in two separate studies among pregnant women [69], however, our results were higher than $0.6 \%$ among pregnant women in Norway [70].

In the four studies performed in blood donors, the WP was $17.03 \%$ among 1123 cases. This prevalence is also closer to the general population prevalence, despite that each one of them was carried out with different diagnostic tests (one with SF [46], one not reported [47], one with IFAT [5] and one with ELISA, respectively [48]).

Regarding the blood donors group, this group has a lower WP when compared to the general population. Transfusion Medicine guidelines have pronounced that donated blood should be screened for toxoplasmosis, because of the potential risk for blood receptors receiving transfusions from subjects in the acute phase of infection. Therefore, testing of toxoplasmosis in blood donations should be mandatory in the country [5,46-48].

Three studies performed in Mexico were in women with miscarriages having primary infection during pregnancy. There were clear differences between the WP of pregnant women $15.62 \%$ whereas those with miscarriages had 35.96\% ( $\mathrm{p}<0.05)$. These results are concordant with other studies that have shown an association between high prevalence of miscarriages and Toxoplasma infection $[39,44,45]$.

Another risk group was the immunocompromised patients. Among 627 cases, the WP of $20.2 \%$ was the 
same as in the general population. One explanation is that the patients were diagnosed by different methods, and two studies performed in Durango had the lowest prevalence [47,49-52].

The highest WP was $37.24 \%$ in the mentally-ill patients, at least $18.27 \%$ higher than the general population [55]. Several authors have reported a high prevalence of Toxoplasma antibodies in patients with schizophrenia, although other factors such as genetic may be present in the schizophrenia, different reports have shown that Toxoplasma is somehow associated with cases of schizophrenia. This finding justifies the need to examine the relationship between toxoplasmosis and schizophrenia with pre-clinical and clinical trials aimed to improve prevention and treatment programs in patients with psychiatric illness $[71,72]$. On the other hand, a metaanalysis of latent Toxoplasma gondii infection in immunocompetent hosts and cryptogenic epilepsy showed a strong association between seroprevalence rates for toxoplasmosis and prevalence rates of epilepsy. If an etiological connection can be proven, it would have implications for the implementation of prevention and treatment strategies for Toxoplasma disease [73].

Cat owners and slaughterhouse workers presented both a CP and WP of $21.88 \%$ each. This may be because only five studies with 501 individuals with this attribute were analyzed. Regarding patients with nonrelated comorbidity, WP was $12.28 \%$, lower than in the general population. Three groups were studied with STA and four more from Durango had the lowest prevalence in the country, which may show that their comorbidity was not related to the risk of infection.

Another important factor is the difference in the prevalence of $T$. gondii infections due to the sensitivity and specificity of diagnostic tests, since there are several methods to identify and evaluate antibodies in individuals who were infected by the parasite. Over time, at least four different diagnostic assays have been used worldwide that range from the lowest specificity and sensitivity like the STA progressing on to the SF Dye Test and other similar tests up to the improved ELISA (Table 2) [7,74].

In regards to the analysis of the study groups adjusted by risk factors (with or without) and diagnostic test, we found that only those tested by ELISA showed a WP of $17.66 \%$ in the risk factor groups against a WP of $8.92 \%$ in the groups without risk factors. However, with the other methods, both crude and weighted prevalence were reduced; however, the number of studied individuals was higher. This analysis demonstrates that the prevalence varies according to the diagnostic method and by the number of individuals tested in each study group (Figure 2).

The epidemiological behavior of Toxoplasma infection showed a negative slope of $-0.1 \% /$ year, which represented an accumulated decrement of nearly $6 \%$ in the prevalence of infection in Mexico after 60 years. This may suggest that we have not paid enough attention to $T$. gondii infection as a public health problem though it tends to decrease. However, another key issue is that if the studies carried out in the first 10 years had been tested with ELISA then the decrement would have been even lower (Figure 2).

In this study, stratification of studies based on molecular assays was not feasible because none was reported. However, genotyping has been reported in other Latin American populations, such as Colombia where a virulent strain ( $\mathrm{LD}_{100}$ of 10 tachyzoites) was identified as clonal type 1 (CIBMUQ/HDC) [75]. Additionally, in another study, the GRA6 type I/III profile was the most frequent among asymptomatic cases $(68 / 148,45.9 \%)$ and in severe multi visceral cases $(2 / 4,50 \%)$. Furthermore, GRA6 type II, was found in one case of congenital toxoplasmosis, one case of severe multi visceral infection, one case of ocular infection, and in five cases $(5 / 148,3.4 \%)$ of asymptomatic patients [76]. Further studies based on genetic-based diagnostic assays will be relevant in the future, since Toxoplasma gondii isolates from Latin America have mixed/ recombinant genetic structure.

A 6\% decrement in the prevalence of Toxoplasma infection after 60 years of studies was detected. This decrement is quite low after a relatively long period, contrary to what has been reported in the United States, with a $14 \%$ decrement in only one decade [77]. Therefore, our data warrants that researchers must pay more attention to this disease and to communicate to the medical community the need of improvement of the prevention strategies, together with effective diagnostic testing and management of patients in high risk of infection such as immunocompromised patients and pregnant women.

Further research is still required to understand and clarify the role of $T$. gondii in its diverse routes of transmission, as well as to design better control measures that focus on minimizing the risk of infection. The purpose of such studies should be to aid in the monitoring of changes in the epidemiology of T. gondii infection, and to strengthen educational efforts in order to avoid the transmission of infection in populations that cannot be controlled by drugs alone.

\section{Implications for research}

A crucial factor is the difference in the prevalence of T. gondii infections due to the sensitivity and specificity of the diagnostic tests, since there are several methods to identify and evaluate antibodies in individuals who were infected by the parasite. At least four different diagnostic assays have been used in this study, that range from the lowest specificity and sensitivity like the $\mathrm{CF}$, progressing 
on to the SF dye test and other similar tests up to the improved ELISA.

\section{Conclusion}

In conclusion, to the best of our knowledge, this is the first study that provides a comprehensive view of the epidemiological situation on the prevalence of $T$. gondii infection among the adult Mexican population. It provides not only epidemiologic evidence relevant to Mexico, but to other countries in the Americas and worldwide as well, where it has been documented that $T$. gondii prevalence is shifting, related to regional climate changes among other factors. In this study, the major risk groups with Toxoplasma infection were women with miscarriages, immunocompromised patients, mentally-ill patients and other risk groups. Noteworthy was the psychiatric patients group, since T.gondii can cause serious damage to the central nervous system.

\section{Competing interests}

The authors declare that they have no competing interests.

\section{Authors' contributions}

MLG conceived and designed the research, RT performed statistical tests, MLG and SR wrote the manuscript, CC and RB database information search. All authors read and approved the final version of the manuscript.

\section{Author details}

'Department of Physiology, Neurophysiology Laboratory, Health Sciences University Center, University of Guadalajara, Sierra Mojada \# 950 Edificio N Col. Independencia, Guadalajara, Jalisco 44320, México. "2"Federico Gómez" Children's Hospital of Mexico, Mexico City, Mexico. ${ }^{3}$ Department of Molecular Biology in Medicine, Civil Hospital of Guadalajara "Fray Antonio Alcalde", University of Guadalajara, Hospital \#278, Guadalajara, Jalisco 44280, Mexico.

Received: 10 August 2012 Accepted: 27 October 2012

Published: 26 November 2012

\section{References}

1. Galván-Ramírez ML, Mondragón-Flores R: Toxoplasmosis humana. Guadalajara Jalisco Mexico: Editorial Cuellar; 2001.

2. Galván-Ramirez ML, Madriz A, Rico C, Luna-Pastén H, Rodríguez Pérez LR, Rincón-Sánchez AR, Franco R, Salazar-Montes A, Correa D: Frequency of Toxoplasma gondii in pork meat in Ocotlán, Jalisco, Mexico. J Food Prot 2010, 73:1121-1123.

3. Galván-Ramírez ML, Castillo-de-León Y, Espinoza-Oliva M, Bojorques-Ramos MC, Rodríguez-Pérez LR, Bernal Redondo R, Cañedo-Solares I, Espinoza López L, Correa D: Acute infection of Toxoplasma gondii and cytomegalovirus reactivation in a pediatric patient receiving liver transplant. Transpl Infect Dis 2006, 8:233-236.

4. Galvan-Ramirez ML, Rincon A, Bernal R: Diagnostic of opportunistic parasites in liver transplantation. In Parasitology research trends. Volume 1. 1st edition. Edited by de Bruyn O, Stephane P. New York: Nova; 2010:210-254.

5. Galván-Ramírez ML, Covarrubias X, Rodríguez R, Troyo R, Alfaro N, Correa D: Toxoplasma gondii antibodies in Mexican blood donors. Transfusion 2005, 45:281-282

6. Galván-Ramírez ML, Orozco C, Mancilla J: Seroepidemiology of Toxoplasma gondii in workers of slaughterhouse in Zapopan, Jalisco [abstract]. Int J Infect Dis 2008, 12:384.

7. Galván-Ramirez ML, Sánchez G, Vielma M, Soto Mancilla JL: Presence of anti- Toxoplasma antibodies in humans and their cats the urban zone of Guadalajara. Rev Soc Bras Med Trop 1999, 32:483-488.

8. Patz J, Graczyk T, Geller N, Vittor AY: Effects of environmental change on emerging parasitic diseases. Int J Parasitol 2000, 30:1395-1405.

9. Meerburg BG, Kijlstra A: Changing climate-changing pathogens: Toxoplasma gondii in North-Western Europe. Parasitol Res 2009, 105:17-24.
10. Tenter AM, Heckeroth AR, Weiss LM: Toxoplasma gondii: from animals to humans. Int J Parasitol 2000, 30:1217-1258.

11. el-On J, Peiser J: Toxoplasma and toxoplasmosis. Harefuah 2003, 142:48-55.

12. Galvan-Ramirez ML, Troyo-Sanroman R, Roman S, Bernal-Redondo R, Vazquez-Castellanos $\mathrm{JL}$ : Prevalence of toxoplasma infection in Mexican newborns and children: a systematic review from 1954 to 2009. ISRN Pediatr 2012, 201 (2):501216. doi:10.5402/2012/501216.

13. Guex-Crosier Y: Update on the treatment of ocular toxoplasmosis. Int $\mathrm{J}$ Med Sci 2009, 6:140-142.

14. Balaskas K, Vaudaux J, Boillat-Blanco N, Guex-Crosier Y: Azithromycin versus Sulfadiazine and Pyrimethamine for non-vision-threatening toxoplasmic retinochoroiditis: a pilot study. Med Sci Monit 2012, 18:96-302

15. Palomino F, Soto R, Villegas L: Un caso de toxoplasmosis. Bol Hosp Infant Mex 1950, 1:24-39

16. Biagi F: Cutirreaciones con Toxoplasmina en Tampico. Rev Med Hosp Gen Mex 1951, 14:191.

17. Bustos C, Aguilar C, López V: Investigación sobre toxoplasmosis en la Región de Orizaba, Ver. Boletín de Salubridad e Higiene 1952, 8:1-18.

18. Biagi F: Intradermal tuberculin and toxoplasmin reactions in Escarcega, Camp. transmission of toxoplasmosis. Med Rev Mex 1953, 33:268-272.

19. Varela G, Martínez E, Treviño A: Toxoplasmosis en la República Mexicana. Rev Inst Salubr Enf Trop 1953, 13:217-224.

20. Varela $G$, Roch E, Vázquez A: Virulence, culture, polysaccharides, toxins and the dye test, studies with a strain of Toxoplasma gondii. Rev Inst Salubr Enferm Trop 1955, 15:73-80.

21. Biagi F, Alemany J: Intradermo-reacciones con Toxoplasmina en Ixtapala, D.F. Bol Med Hosp Inf Mex 1957, 14:125-128.

22. Varela G, Roch E, Zavala J: Estudios sobre toxoplasmosis en México. Salud Pub Mex 1961, 3:451-454.

23. Roch E, Varela G: Diversos aspectos de la investigación sobre toxoplasmosis en México. Resultados obtenidos en 29,883 reacciones de Sabin y Feldman efectuadas de 1953 a 1965. Rev Invest Salud Publica 1966, 26:31-49.

24. Goldsmith RS, Kagan I, Reyes-Gonzalez G, Cedeño MA, Ferrerira J: Seroepidemiologic studies in Oaxaca, Mexico. Bull Pan Am Health 1972, 6:39-52.

25. Galván M, Garzón M: Estudio serológico en niños con Toxoplasmosis. Rev Latinoam Microbiol 1989, 31:267-270.

26. Goldsmith R, Kagan I, Zárate R, Reyes-González MA, Cedeno-Ferreira J: Low Toxoplasma antibody prevalence in serologic surveys of humans in southern Mexico. Arch Invest Med Mex 1991, 22:63-73.

27. Velasco-Castrejón O, Salvatierra-Izaba B, Valdespino JL, Sedano-Lara AM, Galindo-Virgen S, Magos C, Llausás A, Tapia-Conyer R, Gutiérrez G, Sepúlveda J: Serepidemiología de la Toxoplasmosis en México. Salud Publica Mex 1992, 34:222-229.

28. Galván-Ramírez M, Rodríguez R, Ledesma S, Sifuentes LM, Armenta AS, Bayardo D, Ramírez BJ, Troyo Sanromán R: Seroepidemiology of toxoplasmosis in high-school students in the metropolitan area of Guadalajara, Jalisco, Mexico. Sci Med (Porto Alegre) 2010, 20:1-10.

29. Kelso Santos E, Torres E, Cárdenas del Toro C, Salinas MC, Medina CE: Seropositividad a Toxoplasma gondii en adultos del área metropolitana de Monterrey: Reporte preliminar. Med Univer 2000, 2:77-81.

30. Alvarado-Esquivel C, Cruz-Magallanes HM, Esquivel-Cruz R, Estrada-Martínez S, Rivas-González M, Liesenfeld O, Martínez-García SA, Ramírez E, Torres-Castorena A, Castañeda A, Dubey JP: Seroepidemiology of Toxoplasma gondii infection in human adults from three rural communities in Durango State, Mexico. J Parasitol 2008, 94:811-816.

31. Alvarado-Esquivel C, Rojas-Rivera A, Estrada-Martínez S, Sifuentes-Álvarez A, Liesenfeld O, García-López CR, Dubey JP: Seroepidemiology of Toxoplasma gondii infection in a Mennonite community in Durango State, Mexico. J Parasitol 2010, 96:941-945.

32. Alvarado-Esquivel C, Estrada-Martínez S, Pizarro-Villalobos H, Arce-Quiñones M, Liesenfeld O, Dubey JP: Seroepidemiology of Toxoplasma gondii infection in general population in a northern Mexican city. J Parasitol 2011, 97:40-43.

33. Alvarado-Esquivel C, Torres-Castorena A, Liesenfeld O, Estrada-Martinez S, Urbina-Alvarez JD: High seroprevalence of Toxoplasma gondii infection in a subset of Mexican patients with work accidents and low socioeconomic status. Parasit Vectors 2012, 5:13.

34. Alvarado-Esquivel C, Estrada-Martínez S, García-López CR, Rojas-Rivera A, Sifuentes-Álvarez A, Liesenfeld O: Seroepidemiology of Toxoplasma gondii Infection in Tepehuanos in Durango, Mexico. Vector Borne Zoonotic Dis 2012, 12:138-142. 
35. Biagi F, Islas-Pérez M, Gonzalez C: Frecuencia de la toxoplasmosis en relación al parto. Gac Med Mex 1974, 108:127-130.

36. Fernández M, Sibala M, Granier M: Encuesta sero-epidemiológica de anticuerpos anti-Toxoplasma gondii en 125 mujeres embarazadas del oriente del Estado de Tabasco. Bol Med Hosp Infant Mex 1986, 43:274-278.

37. Reyes VM Edl, Machain A, Estrada V, García MP: Toxoplasmosis humana. Ginecol Obstet Mex 1965, 20:749-759.

38. Galván-Ramírez ML, Flores M, Borbas V, Rodriguez R, Sayas M, Hernández SV: Prevalencia de infección por Toxoplasma gondii en mujeres con embarazo de alto riesgo y normal y sus recién nacidos. In Investigaciones en Salud Materno Fetal. 1st edition. Edited by Isabel V, Noe A. Guadalajara, Jalisco Mexico: Universidad de Guadalajara; 2007:235-243.

39. Galván Ramírez M de la L, Soto Mancilla J, Velasco Castrejón O, Pérez Medina R: Incidence of anti-Toxoplasma antibodies in women with high-risk pregnancy and habitual abortions. Rev Soc Bras Med Trop 1995, 28:333-337.

40. Jaramillo P, Pescador S, Galván M, García F: Hábitos higiénicos, tenencia de animales y toxoplasmosis en mujeres embarazadas en la ciudad de Toluca. Asoc Mex de Epidemiología Vet 2003, 1:138-142.

41. Alvarado-Esquivel C, Sifuentes-Alvarez A, Narro-Duarte SG, Estrada-Martínez JH, Díaz-García O, Liesenfeld SA, Martínez-García A, Canales-Molina A: Seroepidemiology of Toxoplasma gondii infection in pregnant women in a public hospital in northern Mexico. BMC Infect Dis 2006, 13:113.

42. Alvarado-Esquivel C, Torres-Castorena A, Liesenfeld O, García-López CR, Estrada-Martínez S, Sifuentes-Alvarez A, Marsal-Hernández JF, Esquivel-Cruz R, Sandoval-Herrera F, Castañeda JA, Dubey JP: Seroepidemiology of Toxoplasma gondii infection in pregnant women in rural Durango, Mexico. J Parasitol 2009, 95:271-274.

43. Cañedo-Solares I, Ortiz-Alegría LB, Figueroa-Damián R, Bustos-Bahena ML, González-Henkel H, Calderón-Segura E, Luna-Pastén H, Correa D: Toxoplasmosis in pregnancy: determination of $\operatorname{lgM}, \lg G$ and avidity in filter paper-embedded blood. J Perinatol 2009, 29:668-672.

44. Roch E: Compendio de Toxoplasmosis. México: Editorial Patria; 1971.

45. Zavala Velazquez JE, Guzman-Marin E, Barrera M, Rodriguez E: Toxoplasmosis y aborto en pacientes del Hospital O'Horan de Mérida, Yucatan. Sal Pub Mex 1989, 31:664-668.

46. Carrillo C: Toxoplasmosis en donadores de sangre. Rev Med Hosp Gen Mex 1962, 25:295-297.

47. Gongora-Biachi RA: Anticuerpos contra Toxoplasma gondii en pacientes con VIH en Yucatan. Rev Invest Clin 1998, 50:419-422.

48. Alvarado-Esquivel C, Mercado-Suarez MF, Rodríguez-Briones A, Fallad-Torres L, Ayala-Ayala JO, Nevarez-Piedra LJ, Duran-Morales E, Estrada-Martínez S, Liesenfeld O, Márquez-Conde JA, Martínez-García SA: Seroepidemiology of infection with Toxoplasma gondii in healthy blood donors of Durango, Mexico. BMC Infect Dis 2007, 13:1-7.

49. Biagi F: Nota sobre toxoplasmino-reacciones en leprosos. Rev Asoc Medica Mex 1952, 32:241-243.

50. Galván-Ramírez ML, Valdez V, Vargas G, Jiménez González O, García Cosio C, Vielma M: Prevalence of IgG e IgM Anti- Toxoplasma antibodies in patients with HIV and acquired immunodeficiency syndrome AIDS. Rev Soc Bras Med Trop 1997, 30:465-467.

51. Alvarado-Esquivel C, Liesenfeld O, Torres-Castorena A, Estrada-Martínez S, Urbina-Alvarez JD, Ramos-de la Rocha M, Márquez-Conde JA, Dubey JP: Seroepidemiology of Toxoplasma gondii infection in patients with vision and hearing impairments, cancer, HIV, or undergoing hemodialysis in Durango, Mexico. J Parasitol 2010, 96:505-508.

52. Alvarado-Esquivel C, Torres-Berumen JL, Estrada-Martínez S, Liesenfeld O, Mercado-Suarez MF: Toxoplasma gondii infection and liver disease: a casecontrol study in a northern Mexican population. Parasit Vectors 2011, 4:1-7.

53. Gutiérrez E, Manzano J, Biagi F: Encuesta sobre toxoplasmosis en un grupo de débiles mentales. Rev Ins Sal Enf Trop 1954, 14:197-200.

54. Tay J, Gutierrez Q, Fernandez P, Romero C, Ruiz G, Martinez B: Infección por Toxoplasma gondii en niños con parálisis cerebral infantil. Bol Chil Parasitol 1997, 52:17-21.

55. Alvarado-Esquivel C, Urbina-Álvarez JD, Estrada-Martínez S, Torres-Castorena A, Molotla-de-León G, Liesenfeld O, Dubey JP: Toxoplasma gondii infection and schizophrenia: a case control study in a low Toxoplasma seroprevalence Mexican population. Parasitol Int 2011, 60:151-155.

56. Alvarado-Esquivel $\mathrm{C}$, Liesenfeld $\mathrm{O}$, Márquez-Conde JA, Estrada-Martínez $\mathrm{S}$, Dubey JP: Seroepidemiology of infection with Toxoplasma gondii in workers occupationally exposed to water, sewage, and soil in Durango, Mexico. J Parasitol 2010, 96:847-850.
57. Alvarado-Esquivel C, Liesenfeld O, Estrada-Martínez S, Félix-Huerta J: Toxoplasma gondii infection in workers occupationally exposed to raw meat. Occup Med Lond 2011, 61:265-269.

58. Alvarado-Esquivel C, Liesenfeld O, Márquez-Conde JA, Cisneros-Camacho A, Estrada-Martínez S, Martínez-García SA, González-Herrera A, García-Corral N: Seroepidemiology of infection with Toxoplasma gondii in waste pickers and waste workers in Durango, Mexico. Zoonoses Public Health 2008, 55:306-312.

59. Alvarado-Esquivel C, Estrada-Martínez S: Toxoplasma gondii infection and abdominal hernia: evidence of a new association. Parasit Vectors 2011, 4:1-12.

60. Frenkel JK: Dermal hypersensitivity to toxoplasma antigens. Proc Soc Exp Biol Med 1948, 68:634-639.

61. Sabin BH: Dyes as microchemical indicators of a new immunity phenomenon affecting a protozoon parasite (Toxoplasma). Science 1948, 108:660-663.

62. Warren J, Russ SB: Cultivation of toxoplasma in embryonated egg; an antigen derived from chorioallantoic membrane. Proc Soc Exp Biol Med 1948, 67:85-89.

63. Feltcher S: Indirect fluorescent antibodies technique in the serology of Toxoplasma gondii. J Clin Pathol 1965, 18:193-199.

64. Lunde MN, Jacobs L: Characteristics of the Toxoplasma hemagglutination test antigen. J Immunol 1959, 82:146-150.

65. Kwantes W, Payne RA, Ludlam GB, Bridges JB, Fleck DG: An assessment of a latex agglutination slide test for toxoplasma antibody. J Clin Pathol 1972, 25:359-360.

66. Yehudith N, Goerges D, Remington J: IgM enzyme-linked inmunosorbent assay test for the diagnosis of congenital Toxoplasma infection. J Pediatr 1981, 98:32-36.

67. Roman S, Panduro A, Aguilar-Gutierrez Y, Maldonado M, Vazquez-Vandyck M, Martinez-Lopez E, Ruiz-Madrigal B, Hernandez-Nazara Z: A low steady $\mathrm{HBsAg}$ seroprevalence is associated with a low incidence of HBV-related liver cirrhosis and hepatocellular carcinoma in Mexico: a systematic review. Hepatol Int 2009, 3:343-355.

68. Borenstein M, Hedges LV, Higgins JPT, Rothstein HR: Introduction to metaanalysis. Chichester, West Sussex: John Wiley \& Sons Ltd; 2009

69. Ghoneim NH, Shalaby SI, Hassanain NA, Zeedan GS, Soliman YA Abdalhamed AM: Comparative study between serological and molecular methods for diagnosis of toxoplasmosis in women and small ruminants in Egypt. Foodborne Pathog Dis 2010, 7:17-22.

70. Jenum PA, Stray-Pedersen B, Melby KK, Kapperud G, Whitelaw A, Eskild A, Eng J: Incidence of Toxoplasma gondii infection in 35,940 pregnant women in Norway and pregnancy outcome for infected women. $J$ Clin Microbiol 1998, 36:2900-2906.

71. Dion S, Barbe PG, Leman S, Camus V, Dimier-Poisson I: Schizophrénie et toxoplasmose. Med Sci (Paris) 2009, 25:687-691.

72. Torrey EF, Bartko JJ, Lun ZR, Yolken RH: Antibodies to Toxoplasma gondii in patients with schizophrenia: a meta-analysis. Schizophr Bull 2007, 33:729-736.

73. Palmer BS: Meta-analysis of three case controlled studies and an ecological study into the link between cryptogenic epilepsy and chronic toxoplasmosis infection. Seizure 2007, 16:657-663.

74. Kodym P, Machala L, Roháčová H, Širocká B, Malý M: Evaluation of a commercial IgE ELISA in comparison with IgA and IgM ELISAs, IgG avidity assay and complement fixation for the diagnosis of acute toxoplasmosis. Clin Microbiol Infect 2007, 13:40-47.

75. Gallego C, Gallego C, Castaño JC, Giraldo A, Ajzenberg D, Dardé ML, Gómez JE: Caracterización biológica y molecular del aislamiento CIBMUQ/HDC, una cepa colombiana de referencia para Toxoplasma gondii. Biomedica 2004, 24:282-290.

76. Sousa S, Ajzenberg D, Vilanova M, Costa J, Dardé ML: Use of GRA6-Derived synthetic polymorphic peptides in an immunoenzymatic assay to serotype Toxoplasma gondii in human serum samples collected from three continents. Clin Vaccine Immunol 2008, 15:1380-1386.

77. Jones JL, Kruszon-Moran D, Sanders-Lewis K, Wilson M: Toxoplasma gondii infection in the United States, 1999 2004, decline from the prior decade. AmJTrop Med Hyg 2007, 77:405-410.

\section{doi:10.1186/1756-3305-5-271}

Cite this article as: Galvan-Ramirez et al:: A systematic review and metaanalysis of Toxoplasma gondii infection among the Mexican population. Parasites \& Vectors $2012: 271$. 